\title{
FLUX MAPPING AND MAGNETIC BEHAVIOR OF GRAIN BOUNDARIES IN Nd-Fe-B MAGNETS
}

V.V. Volkov and Y. Zhu

Dept.of Applied Science, Brookhaven National Laboratory, Upton, NY 11973, volkov@bnl.gov

\begin{abstract}
Advanced Fresnel-and Foucault-Lorentz microscopy were applied to analyze magnetic behavior of the grain boundaries in Nd-Fe-B hard magnets. In-situ TEM magnetizing experiments combined with these imaging methods revealed the process of magnetization reversal in polycrystalline sintered and die-upset $\mathrm{Nd}-\mathrm{Fe}-\mathrm{B}$ under various magnetic fields. Fine details of magnetic flux distribution, derived from the magnetic interferograms created by phase-coherent Foucault imaging, provide a quantitative description of the local variation of magnetic flux. Our study suggests that the grain boundaries play an important multi-functional role in the reversal of magnetization, by acting as (a) pinning centers of domain walls, (b) centers of nucleation of reversal domains, and (c) sinks or sources for migrating magnetostatic charges and/or dipoles. They also ensure a smooth transient for irreversible remagnetization in polycrystalline samples.
\end{abstract}

\section{INTRODUCTION}

Anisotropic Nd-Fe-B hard magnets have received considerable scientific attention because of their importance in the technology of permanent magnets. The range of the applications is expected to grow rapidly as their properties and cost-effectiveness are improved. Although the high remanence of anisotropic permanent magnets is known to be closely related to grain texture and alignment, the coercivity mechanisms and their relation to microstructure such as grains size, grain boundary (GB) structure and admixtures of secondary phases are still elusive. Therefore, a nanoscale characterization of $\mathrm{GB}$ properties and their magnetic behavior serves an important step towards the understanding of coercivity mechanisms in anisotropic hard magnets.

Different TEM methods of magnetic imaging, based on Lorentz microscopy principles, have been developed recently to analyze magnetic materials. However, only a few of them are quantitative and can be applied in real time, which is crucial for addressing magnetization reversal mechanisms in Nd-Fe-B magnets. A unique tool may be the novel phase-coherent Foucault (PCF) microscopy $[1,2]$. This method does not require any special attachment such as a bi-prism in electron holography. The PCF-images can be acquired in any TEM with a coherent source. They display in-plan local distribution of magnetic flux. If the variation of sample thickness is negligible, the PCF-images can be easily converted to a local induction map.

Another purpose of our work was to demonstrate how in-situ TEM magnetizing experiments reveal GB magnetic behavior in Nd-Fe-B magnets under a variable external field. We demonstrate that dynamic magnetic imaging, combined with microstructure analysis, can yield a better understanding on how magnetic behavior of GBs might be related to coercivity of Nd-Fe$B$ magnets.

\section{EXPERIMENTAL}

Two types of anisotropic hard magnets were used: sintered $\mathrm{Nd}_{15} \mathrm{Fe}_{78.5} \mathrm{~B}_{6.5}$ and die-upset $\mathrm{Nd}_{13.75} \mathrm{Fe}_{80.25} \mathrm{~B}_{6}$. The nominal compositions of the samples had a slight excess of $\mathrm{Nd}$ over the stoichiometry of $\mathrm{Nd}_{2} \mathrm{Fe}_{14} \mathrm{~B}$ and both had high energy-products. Their fabrication procedures were published in [3,4] and [5-8], respectively. In-situ TEM was carried out using a JEM FEG 
$3000 \mathrm{~F}$ microscope under the free-lens control mode. The internal magnetic field $(0.02 \sim 3 \mathrm{~T})$ of the TEM was carefully calibrated by SQUID [9] and Hall probe [10] measurements.

\section{RESULTS AND DISCUSSION}

\section{A. Magnetic flux mapping}

It is essential that a method we use for image analysis of local domain structure of magnetic material is a simple, reliable and quantitative. The novel approach based on magnetic interferograms, created by PCF-imaging, complies with most of these demands. Recently, PCF microscopy was applied for induction mapping of closure domains in soft Fe- and Co-films [1, 2]. In the present work, we modified this method to analyze the hard magnets. Fig.1 illustrates the principles of magnetic-flux observation and PCF-interferograms. We assumed that all the interactions of electron waves with a sample could be explained by their interactions with electromagnetic fields. Then, from Schrödinger equation the phase shift $(\Delta \theta)$ between the two electron beams $\Psi=\mathrm{R} \exp (\mathrm{i} \theta)$, where $\theta=\varphi / \hbar$, can be defined as [1]

$$
\Delta \theta=\Delta \varphi / \hbar=(\sqrt{2 m e} / \hbar) \oint \sqrt{V} d s-(e / \hbar) \oint \mathbf{A} d s=(\sqrt{2 m e} / \hbar) \oint \sqrt{V} d s-(e / \hbar) \int \mathbf{B} d s,
$$

Here, $\mathrm{m}$ and $\mathrm{e}$ are the electron mass and charge, and $\hbar=\mathrm{h} / 2 \pi$ is the Planck's constant. V and $\mathbf{A}$ are the scalar and vector potentials (div $\mathbf{A}=0$ ), representing the electrostatic and magnetic field contributions. The contour integral of $\mathbf{A}$ is taken for a closed path along two electron trajectories, and the integral of $\mathbf{B}$ is performed for the normal component of flux density $\left(B_{n}\right)$ over the surface enclosed by the two electron paths.
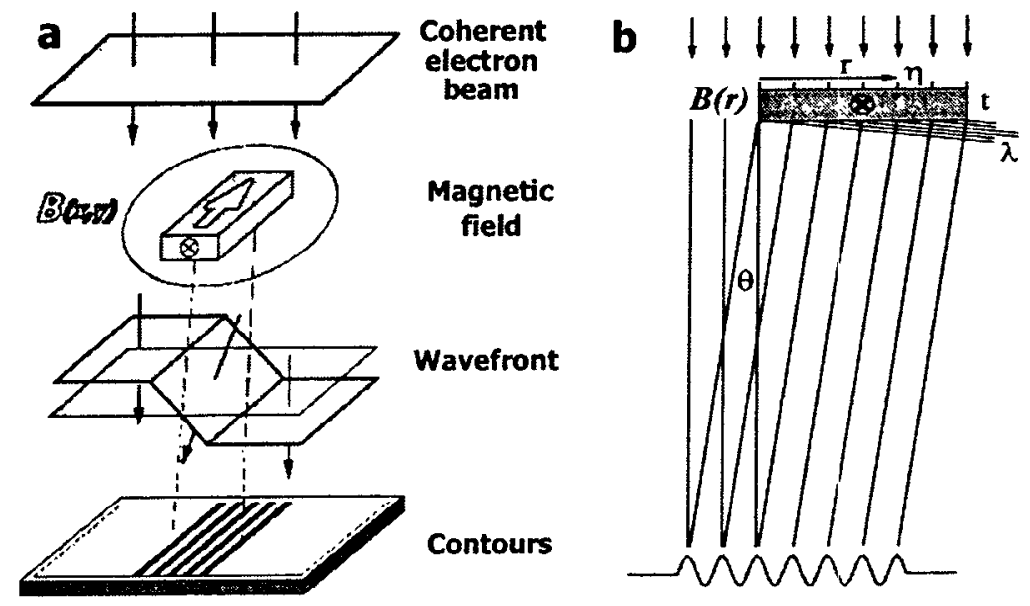

Fig. 1 (a) Principle of magnetic-flux observation, and (b) phase-coherent Foucault imaging.

In FEGTEM, the fringe pattern (interferogram) formed by the phase shift between intersecting coherent electron waves can be well described by the Eq.(1), which can be reduced to the following one, assuming a uniform film thickness $(t)$ and absence of electrostatic stray field:

$$
\Delta \varphi / \hbar=(\sqrt{2 m e V} / 2 \hbar) \cdot\left(V_{0} / V\right) \cdot t+(e / \hbar) \int \mathbf{B} \cdot d \mathbf{s} \approx C \cdot V_{0} \cdot t+(e / \hbar) \cdot B_{n} \cdot \Delta S
$$

where $\mathrm{V}_{0}$ is the inner potential. It is assumed here that $\mathrm{V}_{0} \approx 20 \mathrm{~V}, \mathrm{~V}_{0} \ll \mathrm{V}=300 \mathrm{kV}$ and $C=\sqrt{m e / 2 V}$ is a constant. The first term in Eq.(2) accounts for the contribution of film thickness to phase shift, the second is known as the Aharonov-Bohm shift [11]. Assuming that $\mathrm{B}$-field is available only in the film, the integral in Eq.(2) transforms to $\Delta \Phi(x, y)=B_{n} \Delta S$, where $\Delta S=(r \cdot t)$, as shown in Fig. 1b. When two electron beams enclose an elementary flux $d \Phi=$ 
$h / e$, their phase shift in Eqns.(1-2) due to the magnetic field reaches $\Delta \theta=2 \pi$, which gives the maximum fringe intensity in the interferogram.

A similar result can be derived from a classical approach by taking into account the deflection angle $\theta$ of the electron beam due to the action of Lorentz force by magnetic domains:

$$
\sin \theta \approx \theta=e \cdot B_{n} \cdot t / m v=e \cdot B_{n} \cdot t \cdot \lambda / h
$$

where $\mathrm{v}$ and $\lambda$ are the velocity and wavelength. The Lorentz deflection angle of the electron beam is very small $\sin \theta \approx \theta=4 \cdot 10^{-5} \mathrm{rad}$ in our TEM experiments (electron wavelength is $\lambda=$ $0.02 \AA$ at $300 \mathrm{kV}$ ). The second necessary equation we derive from the interference maxima condition (Fig. 1b) as

$$
\eta \cdot \sin \theta=\lambda
$$

where $\eta(x, y)$ is the fringe spacing, corresponding to local $2 \pi$-phase shift between the coherent electron beams. It follows from (3) and (4) that for a single fringe spacing $\eta$ we find

$$
D_{\eta}(x, y) / t=1 /(\eta \cdot t)=(e / h) \cdot B_{n}(x, y)
$$

where $D_{\eta}=1 / \eta$ is a fringe density (number of fringes per unit surface area). Further from Eq.(5), for the $\mathrm{n}$-th interference maximum with coordinate $r(x, y)=n \cdot \eta$ (n-integer) the expression for magnetic flux transforms simply to

$$
\Delta \Phi=B_{n} \cdot \Delta S=B_{n} \cdot(n \eta \cdot t)=B_{n} \cdot\left(n \frac{h}{e \cdot B_{n} \cdot t} \cdot t\right)=n \cdot \frac{h}{e}
$$

where $h / e-$ is a single quantum of magnetic flux, corresponding to each fringe spacing.

Eqns.(5-6) open a very effective, easy way for interpretation and nanometer scale mapping of magnetic flux $\Phi(\mathrm{x}, \mathrm{y})$ on the basis of PCF-images, as we can visualize a single fluxon $d \Phi=$ $h / e=4 \cdot 10^{-15} \mathrm{~Wb}$ (Eq.6) flowing between two adjacent contour lines. A PCF-image thus provides direct quantitative flux mapping $\Phi(\mathrm{x}, \mathrm{y})$. The vector of magnetic flux density $B_{\mathrm{n}}(\mathrm{x}, \mathrm{y})$ follows the local fringe lines direction and its local amplitude is defined by the ratio of local fringe density to a film thickness as $D_{\eta}(x, y) / t=1 /(\eta t)=(e / h) B_{n}(x, y)$ (Eq.(5)). Hence, the fringe density map normalized to film thickness $D_{\eta}(x, y) / t(x, y)$ gives a direct quantitative $B_{n}(x, y)$ inductance mapping .

Fig. 2 shows the experimental in-focus PCF-image of $\mathrm{Nd}_{2} \mathrm{Fe}_{14} \mathrm{~B}$. It contains much more magnetic and structural information then an out-of-focus Fresnel image. Under a properly collimated illumination angle of $4 * 10^{-7} \mathrm{rad}$, the spatial coherence length of $\leq 5 \mu \mathrm{m}$ ("the width of interferogram") can be achieved at $300 \mathrm{kV}$. The black and white contrast of magnetic domains here is similar to one created by non-coherent Foucault imaging. It shows the opposite components of their magnetization. As mentioned above, the interferogram in Fig. 2 directly represents a map of magnetic flux $\Phi_{1}(\mathrm{x}, \mathrm{y})$ for the white-contrast domains, provided the thickness variations of the film are sufficiently small $(\Delta \mathrm{t}<60 \mathrm{~nm})$ to cause an additional $2 \pi$ phase shift in the fringe pattern. Similar interferogram, $\Phi_{2}(x, y)$, can be obtained for black domains after switching the illumination conditions to complementary ones. Superposition of $\Phi_{1}+\Phi_{2}=\Phi(x, y)$ creates a complete map of magnetic flux in a sample. The fringe spacing, directly measured from Fig. 2, yields $\eta=43 \mathrm{~nm}$. By taking into account the magnetic moment of single domain in $\mathrm{Nd}_{2} \mathrm{Fe}_{14} \mathrm{~B}\left(B_{n} \approx I_{\mathrm{s}}=1.6 \mathrm{~T}\right)$ we get from Eq.(5) a reasonable estimated film thickness $\mathrm{t} \approx 60 \mathrm{~nm}$. It suggests that from $1 \pi$-phase shift criteria the spatial resolution of typical magnetic mapping by PCF-technique is about $\sim 20 \mathrm{~nm}$ and may be a function only of the film 


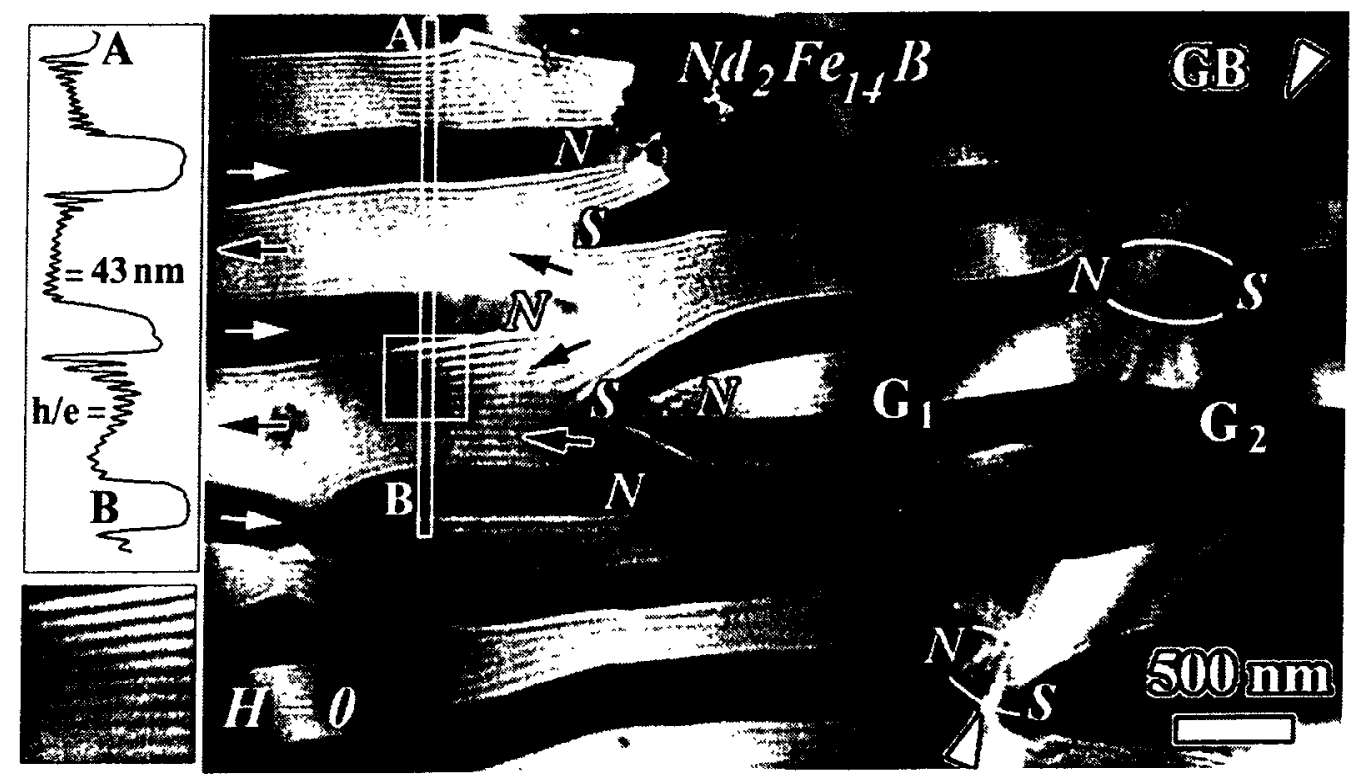

Fig. 2 Phase-coherent Foucault image (magnetic interferogram) of NdFeB-magnet near the grain boundary area. The density of fringes and their directions are directly associated with magnetic flux density $B_{n}(x, y)$ distribution. The insets show the intensity profile along A-B line.

thickness. We note that fringes in Fig. 2 disappear at the domain tips, where the magnetic flux leaves the sample and creates the magnetic poles ( $\mathrm{N} / \mathrm{S}$ charges). These charges concentrate near the GB like simple N/S dipoles or form the metastable multipole's configurations ...N/S/N/S... running across the domain structure. In both cases, it decreases the magnetostatic energy. As a result, a complex magnetic-field distribution appears near the GB (Fig. 2). Notice that for real closure domains, the number of fringes (n) within any fringe contour should remain constant because of the absence of magnetic flux dissipation.

\section{B. In-situ TEM magnetizing experiments}

The nanoscale magnetic behavior of grain boundaries and the magnetization reversal in dieupset and sintered magnets were found to be complex and ruled by different mechanisms.

Die-upset (DU) magnets. Many general features of the grain boundary magnetic behavior can be derived directly from magnetic sensitive conventional Foucault images as shown in Fig. 3

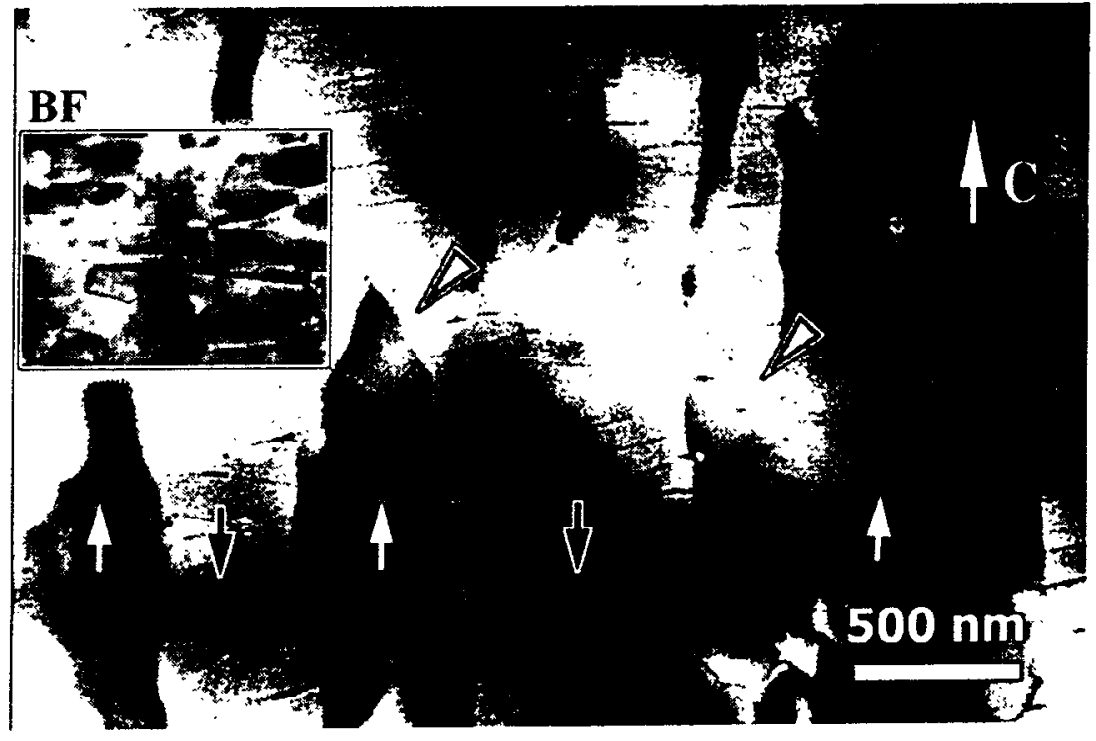

Fig.3 Non-coherent Foucault image of large "interacting /cluster" domains, running across the platelet-like grain texture in DU-magnet along the easy (die-upset) direction marked with c-arrow, showing remanence state of DUmagnet after saturation along hard direction. The local polarization of magnetic domains is marked by small black and white arrows, and the pinning centers by arrowheads. Inset is a brightfield image. 
Notice that the tips of domains with opposite magnetization are often pinned by defects or locally misaligned grains of $\mathrm{Nd}-\mathrm{Fe}-\mathrm{B}$ hard magnetic phase. A few very small reversal domains were observed in remanent state after magnetization of DU-magnet along the easy magnetic (or die-upset) direction. Their presence is associated with the local imperfections, such as the secondary phase inclusions or large-angle GBs, similar to those shown in Fig.3. In-situ TEM magnetizing experiments confirmed that small reversal (or negative) domains, associated with non-aligned grains, continue to grow slowly under the increasing negative field until they nucleate a c-axis aligned reversal domain at the nearest GB. The magnetization reversal in a DU-magnet then transforms rapidly to an irreversible process, until only the pinning of the domain walls at GBs controls the further expansion of reversal domains as shown by successive images (Fig. 4a) captured on video and recorded at different points of the magnetic hysteresis loop (Fig.4b). Thus, the leading mechanism in magnetization reversal of DU-magnets seems to be the nucleation of reversal domains, preferentially at misaligned GBs and interfaces or sample surface where the demagnetizing field is the largest. Fig.4 shows also an example of strong pinning/trapping center (Fig.4a, Frame-3) not vanished at strong negative field $\mathrm{H}<<-\mathrm{H}_{\mathrm{c}}$ (Fig.4b) and its structure model (Fig.4c). This center was found to be responsible for the nucleation of positive domains at $\mathrm{H} \geq+\mathrm{H}_{\mathrm{c}}$ for the 4-th quadrant of hysteresis loop (frames 4-6, Fig.4a), starting from negative magnetization. The presence of a nonmagnetic "pocket" phase at the GBs $[12,13]$ is the necessary condition for "strong pinning" by such a defect center. It reduces the high density of magnetic charges (Fig.4c) by spreading them around this GB-buffer layer.

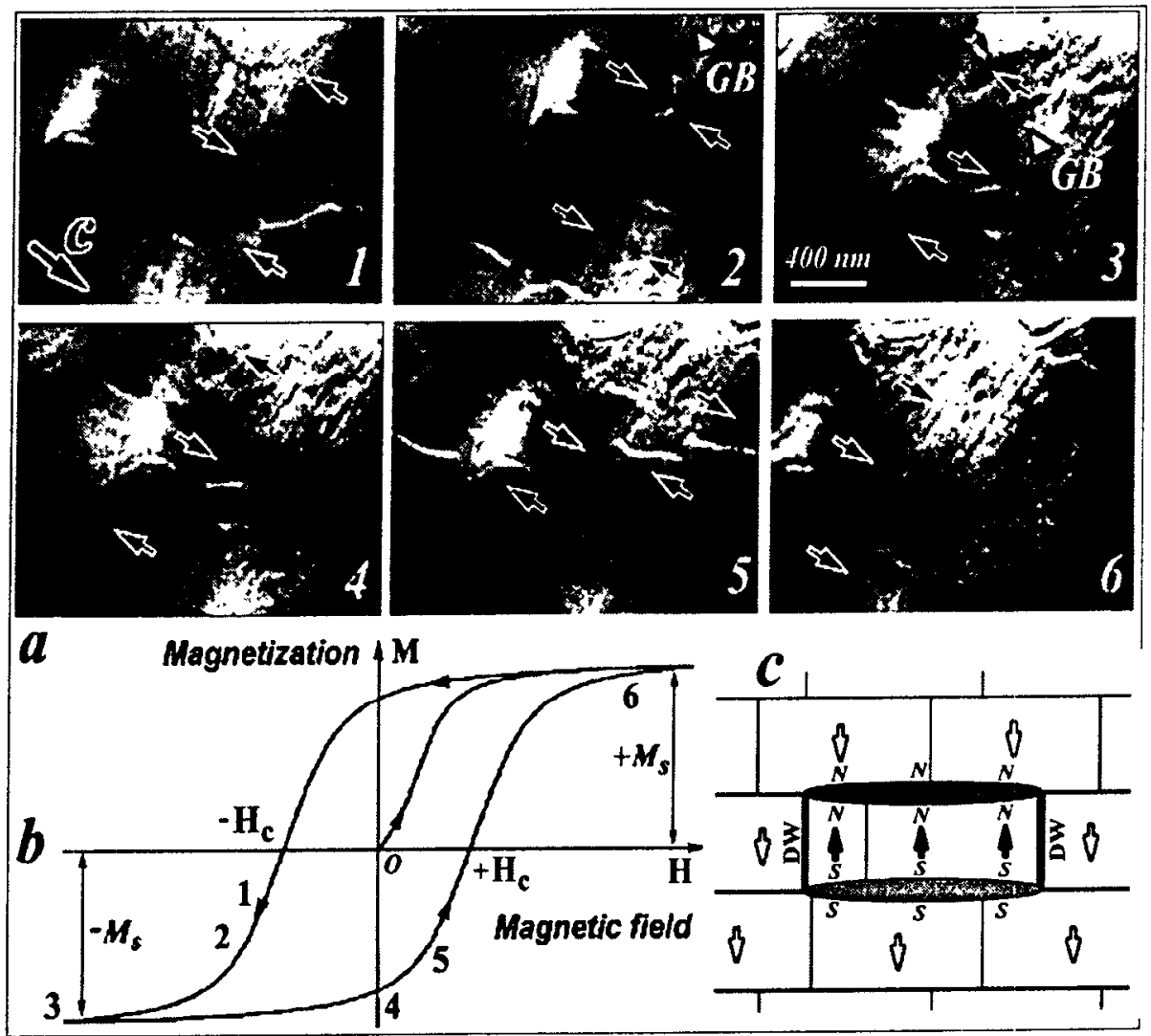

Fig.4 Successive Fresnel images (a) of domain structure in DU-magnet taken at different points (1-6) of the hysteresis loop (b) of the Nd-Fe-B hard magnet.

Magnetization directions of local domains are marked by small arrows and the fine line-contrast perpendicular to these arrows are the grain boundaries.

(c) Structural model showing the origin of strong pinning/trapping center (fig. a: Frame-3) not vanished at strong negative field $\mathrm{H} \leq-\mathrm{H}_{\mathrm{c}}$ (fig. b: point-3). 
Sintered (SI) magnets. Our in-situ TEM observations revealed that the irreversible stage of remagnetization in SI-magnets is often associated with GBs. Fig.5 serves an example showing a domain-splitting mechanism responsible for the reverse domain nucleation near the GBs. It is an essentially irreversible process - the nucleation of negative domains takes place via several sudden splitting of positive domains (Fig.5). Here, a positive domain (marked with black arrow) splits at the GB into "positive-negative-positive" domains under a moderate negative magnetic field. The newly formed domain configuration encloses a pair of new $180^{\circ}$-domain walls, which facilitates further remagnetization of magnet by a simple slow motion of the domain walls.
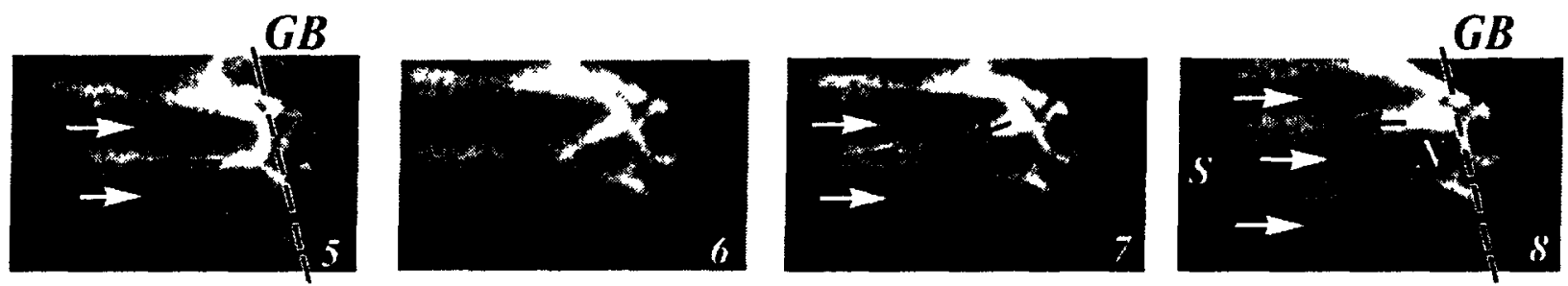

Fig.5 Successive images (5-8) recorded from video showing reversal domain nucleation at the GB in $\mathrm{Nd}-\mathrm{Fe}$-B SI-magnet via splitting of domain with opposite magnetization.

On the other hand, this process may be considered as a cascade-like discharge of magnetic poles (or charges) at the GBs. The domain-splitting mechanism can well explain some experimental observations. For instance, the fact that high concentration of secondary phases in SI-magnets cannot greatly improve the coercivity is because that the classical mechanism of domain wall pinning by defects is no longer the only mechanism for magnets with high density of domain walls.

\section{ACKNOWLEDGEMENT}

This research was supported by the U.S. Department of Energy, Division of Materials, Office of Basic Energy Science, under the Contract No. DE-AC02-98CH10886.

\section{REFERENCES}

[1] A. Tonomura, Surf. Sci. Rep., 20, 317 (1994).

[2] J.N. Chapman, A.B. Johnston, L.J. Heyderman, S. McVitie and W.A.P. Nicholson, IEEE Trans. Magn., 30-6, 4479 (1994); A.B. Johnston, J.N. Chapman, B. Khamsehpour and C.D.W. Wilkinson, J. Phys. D: Appl. Phys. 29, 1419 (1996).

[3] M. Sagawa, S. Fujimura, N. Togawa, H. Yamamoto, Y. Matsuura, J. Appl. Phys. 55, 2083 (1984).

[4] J. Hu , Y. Liu , M. Yin , Y. Wang, B. Hu, Z. Wang, J. of Alloys and Comp., 288, 226 (1999).

[5] R. W. Lee, Appl. Phys. Lett. 46, 790 (1985).

[6] R. W. Lee, E. G. Brewer, N. A. Schaffel, IEEE Trans. Magn. MAG-21, 1958 (1985).

[7] C. D. Fuerst, E. G. Brewer, J. Appl. Phys. 73, 5751 (1993).

[8] V.V. Volkov, Y. Zhu, J. Appl. Phys. 85, 3254 (1999).

[9] V.V. Volkov, D. C. Crew, Y. Zhu, L. H. Lewis, Proc. "Microscopy and Microanalysis", Vol. 5, Suppl. 2, Portland, Oregon, Aug.1-5, 1999, p. 46-47.

[10] V.V. Volkov, Y. Zhu. Private communication.

[11] Y. Aharonov, D. Bohm, Phys. Rev. 115, 485 (1959).

[12] R. K. Mishra, J. Appl. Phys. 62, 967 (1987); R. K. Mishra, Mater. Sci. Eng. B 7, 297 (1991).

[13] V.V. Volkov and Y. Zhu, submitted to J. Magn. Magn. Mater., Nov., 1999. 